\title{
Individualisation sociale et konbini dans le Japon contemporain. La « consommation en réseau » à l'ère informationnelle
}

現代日本のコンビニと個人化社会一情報化時代における「ネット

ワークの消費」一

Individualised Society and Convenience Stores in Japan. Consumption of

Network in the Information Age

\section{Daisuke Tanaka}

Traducteur : Sophie Buhnik

\section{OpenEdition}

\section{Journals}

Édition électronique

URL : http://journals.openedition.org/ebisu/3950

DOI : 10.4000/ebisu.3950

ISSN : 2189-1893

Éditeur :

Institut français de recherche sur le Japon (UMIFRE 19 MAEE-CNRS), Maison franco-japonaise

\section{Édition imprimée}

Date de publication : 23 janvier 2019

Pagination : 133-162

ISSN : 1340-3656

\section{Référence électronique}

Daisuke Tanaka, « Individualisation sociale et konbini dans le Japon contemporain. La

« consommation en réseau » à l'ère informationnelle », Ebisu [En ligne], 56 | 2019, mis en ligne le 24 décembre 2019, consulté le 17 janvier 2020. URL : http://journals.openedition.org/ebisu/3950 ; DOI : 10.4000/ebisu.3950 


\section{Individualisation sociale et konbini dans le Japon contemporain}

$\mathrm{La}$ « consommation en réseau » à l'ère informationnelle

TANAKA Daisuke

現代日本のコンビニと個人化社会

一情報化時代に扮ける「ネットワークの消費」一

田中大介

Individualised Society and Convenience Stores in Japan.

Consumption of Network in the Information Age

TANAKa Daisuke

\Mots-clés : Convenience store, konbini, réseau, individualisation, consommation, informatisation, travailleurs étrangers

L'auteur : Tanaka Daisuke est maître de conférences à l'université féminine du Japon (Nihon joshi daigaku). Ses thèmes de recherche concernent les infrastructures et les mobilités, les équipements dédiés à la consommation et la numérisation des milieux de vie.

Résumé : Cet article analyse la relation entre convenience stores (abrégés ici en konbini) et individualisation dans la société japonaise. En tant que commerces de proximité, les konbini pro- curent un confort sous la forme d'un espace-temps qui repose sur les biens qu'ils vendent, mais aussi sur leur type d'organisation et leurs technologies de l'information. Ils donnent ainsi naissance à un nouveau style de consommation, la " consommation en réseau ". Une socialité ayant des "individus " comme unité principale, s'étend en s'appuyant sur les réseaux de consommation offerts par les konbini. Nous observons que la circulation des individus et des réseaux mise en œuvre grâce à ces magasins japonais modernes définit les limites et l'intérieur de la société que l'on qualifie de japonaise en référence à deux " extérieurs ", l'Amérique et l'Asie. 
】キーワード

コンビニエンスストア、コンビニ、

ネットワーク、個人化、消費、情報化、 外国人労働者

\section{著者}

田中大介：日本女子大学人間社会学部准教授。 研究テーマは、交通機関、消費施設、情報環 境などを通じて構成される都市インフラや モビリティ。

\section{要旨}

本論文では、日本社会におけるコンビニエン スストア（以下、コンビニと省略）と「個人
化社会」の関係を分析する。コンビニという 小売形態は、商品のみならず、その組織形態 や情報技術が可能にする社会的な時間や空 間のかたちを利便性として提供することで、 「ネットワークの消費」という新たな消費の スタイルを生み出した。現代日本における「個 人」を単位とする社会生活は、コンビニとい う「消費のネットワーク」に依存することで 拡大することになる。こうした現代日本のコ ンビニが作り出す「個人とネットワーク」の 循環は、アメリカやアジアという二つの「外 部」を参照しながら、「日本」という社会の 外縁と内包を作り出していることを指摘する。

and space which not only their goods, but also their organization type and information technologies make possible. They thus give birth to a new style of consumption, networked consumption. Social life that has "individuals" as its main unit will expand by relying on the "consumption networks" formed by konbini. We point out that the circulation of individuals and networks produced by these modern Japanese convenience stores defines the outer edges and interior of the society called Japanese, by referring to the two "outsides" of America and Asia.
Abstract: This paper analyses the relationship between convenience stores (konbini) and individualisation in contemporary Japanese society. As a retail form, konbini provide convenience in the form of a time 


\section{Individualisation sociale et konbini dans le Japon contemporain}

$\mathrm{La}$ « consommation en réseaux » à l'ère informationnelle TANAKA Daisuke*

Les commerces de détail qui, sur de petites surfaces, concentrent une gamme diversifiée de services à la personne et la vente d'articles dernier cri et de première nécessité, couramment appelés konbini ${ }^{1}$, se sont répandus dans tout le Japon ${ }^{2}$. Sans se soucier du regard des autres, on peut s'y arrêter au gré

1. En raison de leur usage banalisé dans ce texte, les termes konbini et convenience store ne sont plus mis en italique après leur première occurrence. (N.D.T.)

2. Dans leur nomenclature, les statistiques du ministère de l'Économie, du Commerce et de l'Industrie (Keizai sangyōshō 経済産業省, METI [Ministry of Economy, Trade and Industry]) en donnent la définition suivante : "magasin (ou boutique) en libreservice, proposant des aliments et des boissons, dont la surface de vente va de 30 à 250 mètres carrés, ouvrant au moins 14 heures par jour ». Notre article privilégie quant à lui l'usage de l'expression populaire konbini : au-delà de l'acception officielle, il exprime la familiarité entretenue avec cet espace de consommation disponible partout et à toute heure, gommant les différences entre les enseignes. Ce choix est aussi lié aux modalités singulières du développement des convenience stores au Japon.

Cet article est la traduction d'une version remaniée, en accord avec l'auteur, de " Gendai Nihon no konbini to kojinka shakai. Jōhōka jidai ni okeru "nettowāku no shōhin" " 現代日本のコンビニと個人化社会一情報化時代における「ネットワークの消費」一, Nihon joshi daigaku kiyō 日本女子大学紀要 (Japan Women’s University Journal), vol. 26, 2015.

* Maître de conférences à l'université féminine du Japon (Nihon joshi daigaku). 
du hasard et repartir sans avoir rien acheté : ainsi les konbini, en tant que lieux traversés par d'innombrables individus, ont une dimension ubiquitaire telle qu'ils tendent à être considérés comme des infrastructures et des espaces publics constitutifs de la société japonaise contemporaine (Washizu 2008). Le déclin et le vieillissement actuels de la population menant à une pénurie de main-d'œuvre, l'augmentation du nombre d'employés étrangers et la difficulté à garder les konbini ouverts 24 heures sur 24 suscitent des débats importants; mais, au-delà, c'est la question du maintien de leur rôle d'espaces collectifs et d'infrastructures du quotidien qu'il faudrait revisiter.

Depuis les années 1990, des discours critiques sur les convenience stores (CVS) se sont fait entendre à maintes reprises. Par exemple, les konbini seraient une incarnation du capitalisme post-industriel (Asaba 1990; Matsubara 2000), des foyers de délinquance juvénile disloquant les relations familiales, ou bien ils uniformiseraient les atmosphères locales (Miura 2001, 2004). Toutefois, on a souvent remarqué que les CVS sont un type de commerce de proximité dont le développement est spécifique au Japon, ce qui en fait des "magasins typiquement japonais " (Fujimori 1991). On dit par ailleurs que leur popularité provient d'une pratique consistant à acheter fréquemment et en petites quantités du poisson et d'autres aliments frais, notamment à cause du climat chaud et humide (Moriwaki 2006).

Chacune de ces études présente un grand intérêt. Néanmoins, ne voir dans le konbini que la conséquence d'une rationalisation commerciale, liée à l'idéologie capitaliste tardive d'origine nord-américaine, revient à ignorer le rôle joué par le contexte socioculturel japonais. Inversement, les points de vue confinant le konbini à l'expression des traditions et des techniques vernaculaires essentialisent la "communauté imaginée" nippone, selon une expression empruntée à Benedict Anderson. De ce fait, si le fonctionnement sophistiqué des konbini a fait l'objet d'abondantes analyses, le contexte social qui contribue à reproduire leur "japonité " demeure méconnu.

En 2015, plus de 40 ans se sont écoulés depuis que le modèle du CVS a été importé des États-Unis, plus de 30 ans depuis que le groupe Seven-Eleven a inauguré son premier magasin au Japon. Dans l'intervalle, Seven-Eleven Japan est devenu l'actionnaire principal de l'entreprise-mère américaine, Southland Corp. (en 1991), et s'est mué en une société de grande distribution; sous le nom de Seven \& I Holdings (depuis 2005), elle mène aussi ses propres activités financières. En outre, le second plus 
grand acteur de ce marché, Family Mart, a depuis la décennie 2000 ouvert 6196 magasins à l'étranger et près de 7000 au Japon selon des chiffres d'avril 2007 : qualifié de "CVS d'origine japonaise qui a réussi en Asie " (selon le Nihon shokuryō shinbun 日本食糧新聞 [Journal de l'alimentation], édition du 20 septembre 2006), il a connu un développement régional très rapide. Les konbini ne sont donc pas le produit d'une globalisation unilatéralement conduite sous l'influence des États-Unis, mais ils ne se fondent pas non plus sur les seules traditions et coutumes japonaises. Il est dès lors nécessaire de replacer l'essor des konbini au sein d'un environnement national et international en pleine recomposition, et dans une ère de transition vers un capitalisme intensément numérisé (ou informationnel).

C'est pourquoi notre article, à travers son analyse du phénomène des konbini au Japon, cherche à clarifier les mécanismes qui y établissent une "société en réseau " (Castells 1996), caractérisée par sa fermeture, tout en s'ouvrant à [...] l'Amérique du nord et l'Asie. Nous aimerions en particulier interroger le processus par lequel d'innombrables « individus travailleurs-consommateurs", sans verser dans un nationalisme échevelé, reproduisent l'univers culturel japonais par l'intermédiaire des konbini. En d'autres termes, nous nous demandons comment l'adaptation aux attentes des consommateurs, dans un Japon mi-ouvert, mi-fermé aux mobilités et aux migrations, a permis l'expansion d'un réseau commercial « de type japonais » fortement numérisé, à savoir le konbini.

Dans ce texte, la notion de "réseaux de konbini » ou de "konbini (ou commerces) en réseau » se réfère : 1 ) au développement de chaînes de magasins uniformisés, sous l'égide d'une enseigne qui assure leur organisation à une échelle nationale voire globale; 2) au contrôle de l'offre en surface de vente, en produits et en services par des systèmes informatisés perfectionnés. C'est grâce à ces caractéristiques que les konbini construisent un espacetemps flexible, dans le sens où ces systèmes mettent l'accent sur la collecte de données commerciales, incitant à comparer les konbini entre eux, plutôt que sur les spécificités environnementales des lieux où ils s'implantent. Notre appréhension du réseau s'inscrit dans la théorie de la société en réseau de Manuel Castells, succédant à la société industrielle et où l'état social est défini par un mode de développement dit informationnel : «A network is a 
set of interconnected nodes ${ }^{3}$ (Castells, $1996: 71$ ), un ensemble de nœuds interconnectés qui peut aller jusqu'à l'infini. Bien qu'on lui ait reproché d'être vague, abstrait et ambigu, le concept de société en réseau possède une légèreté qui ressemble à la légèreté des konbini en tant qu'infrastructures matérielles. La logistique y est pensée pour que les produits soient remplacés le plus rapidement possible, en s'appuyant sur la vitesse des technologies de l'information. En outre, les similitudes réelles et perçues entre les konbini donnent l'impression d'être " connecté » à d'autres magasins quand on se sert de l'un d'eux, ce qui correspond à la figure du réseau fait de nœuds et de liens multiples.

Notre argumentation repose sur la collecte, depuis la fin des années 1990, de sources primaires et secondaires sur les konbini, ainsi que sur des données d'enquêtes propres interrogeant les interactions entre mobilités, médias et pratiques urbaines. Elle se déroule comme suit. Dans un premier temps (I), nous discutons de la constitution des konbini en un réseau ajusté à l'échelle des individus. Ensuite (II), nous verrons comment le réseau tissé par les konbini structure un espace-temps défini comme un espace des flux à la temporalité atemporelle $e^{4}$. Nous y dévoilerons les rouages grâce auxquels les CVS examinés en (I) retracent les frontières de la société japonaise par rapport à deux "mondes extérieurs » : 1) le style de vie américain et 2) le travail précaire, assuré par des migrants venus majoritairement d'Asie.

3. En anglais dans le texte. (N.D.T.)

4. Nous traduisons ici les concepts de space of flows et timeless time forgés par Manuel Castells, que Tanaka Daisuke mobilise. (N.D.T.) 


\section{La fabrication de « réseaux d'individus » par les konbini : sur les vestiges de la société japonaise}

\section{De l'ancrage familial et local aux individus konbini-compatibles}

Tout d'abord, qu'est-ce qui a permis aux konbini de se constituer en réseaux essentiels à la vie des individus ? En 2016, le prix littéraire Akutagawa a été décerné à Murata Sayaka pour son roman Konbini ningen コンビニ人間5, dont la protagoniste, une femme de 36 ans, exerce un emploi à temps partiel dans un konbini. Dans le regard de l'héroïne, le konbini dessine un lieu où un individu qui n'occupe pas d'emploi régulier dans une entreprise, ne s'enracine pas dans une communauté locale, ni ne forge de relations humaines stables (amicales, amoureuses, familiales), peut cependant sentir qu'il existe - en tant que personne. Le terme individu désigne ici, non pas une identité indépendante qui se construit réflexivement dans sa relation avec autrui, mais plutôt un soi qui se libère en cessant de se questionner sur sa relation aux autres et sur lui-même.

Dans l'espace des konbini, on trouve surtout les moyens de vivre individuellement. Par exemple, la présence d'un konbini permet d'externaliser la finalité principale de la cellule familiale : la reproduction de la vie quotidienne. Si l'on en croit les résultats des recensements nationaux sur le nombre de ménages par type, les familles nucléaires représentaient 60,2 \% du total des ménages japonais en 1980, contre 19,8\% pour les foyers à une seule personne. Mais la part des premiers a diminué à $58,4 \%$ en 2000 , les seconds passant à 27,6\%. De plus, le taux d'individus non mariés parmi les 30-34 ans, de $21,5 \%$ pour les hommes et 9,1\% pour les femmes en 1980 , s'élève respectivement à 45 et $25,4 \%$ en 2000 . Avec la croissance du nombre de personnes seules ou célibataires, de nombreuses tâches domestiques autrefois accomplies par un membre de la famille sont pour ainsi dire déléguées aux konbini.

Bien que chaque magasin soit standardisé, les konbini peuvent, en s'appuyant sur la gamme variée des produits mis en vente et leur rotation rapide, répondre à des besoins individuels diversifiés. Cette diversité et

5. Traduction française par Mathilde Tamae-Bouhon, sous le titre de Konbini, Denoël, 2018; réédité en 2019 par Gallimard Folio sous le titre de La Fille de la supérette. (N.D.T.) 
cette rotation sont soutenues par un circuit de distribution mobilisant les techniques de l'information et de la communication, grâce au système des franchises et à celui des points de vente ${ }^{6}$. Un tel système de distribution réticulaire permet d'identifier les articles les plus et les moins bien vendus, afin de réduire au maximum l'écart entre offre et demande, et de garantir l'approvisionnement en produits frais ou inédits.

Plus particulièrement, les konbini se sont spécialisés dans la vente de plats préparés pour des clients pressés, ayant peu de temps à consacrer aux tâches domestiques, vivant seuls, de sorte qu'ils ont participé à la diffusion de la pratique du "repas en solo". Ajoutons que les CVS sont devenus des " réservoirs de vie quotidienne " s'adaptant aux attentes des individus, car parallèlement à l'achat de produits, ils proposent toutes sortes de services $^{7}$. De fait, lors d'une enquête menée auprès d'étudiants vers la fin des années 1990, à la question "Que représentent pour vous les konbini ?", environ $70 \%$ des répondants de sexe masculin vivant seuls ont déclaré " qu'ils ne pouvaient s'en passer " (contre $30 \%$ des étudiants habitant chez leurs parents et $40 \%$ des étudiantes vivant seules) (Kurihara \& Tomishima $\left.1998: 85^{8}\right)$.

Par ailleurs, on constate que l'usage des konbini ne se réduit pas à la satisfaction des besoins de tous les jours. Selon la même enquête, plus de $40 \%$ des étudiantes logeant chez leurs parents y voyaient un « lieu où l'on peut tuer le temps ", tandis qu'en moyenne $10 \%$ des étudiants (habitant seuls ou chez leurs parents) et des étudiantes (habitant chez leurs parents) considéraient qu'on peut "d'une certaine façon, s’y détendre ". Pour les - nombreux - étudiants vivant en famille, il est probable que le konbini représente une sorte d'échappatoire. Les réponses semi-ouvertes à cette

6. Le texte japonais emploie l'expression anglaise de point of sales. Ils permettent notamment de bénéficier des mêmes offres promotionnelles ou d'utiliser des cartes de fidélité d'un magasin à l'autre. (N.D.T.)

7. Pour Taki Kōji, l'usage diffus des konbini « incorpore les villes dans une partie de la vie privée, qui est la plus petite échelle d'organisation urbaine, et incorpore la vie privée dans la structure de la ville, soit son échelle la plus large" (Taki 1994 : 54).

8. Le questionnaire s'adressait à des étudiant(e)s, mais des résultats comparables ont été obtenus par de nombreuses enquêtes ciblant d'autres catégories de personnes (Mìto jānaru ミートジャーナル [Journal de l'alimentation] 2000; Chūō kenkyūsha 2001; Tsuneki 2005; Takagi 2005; Hirata, Matsutani \& Naitō 2005). 
enquête contiennent encore les descriptions suivantes : " un endroit où se reposer ", " un endroit qui m'attire, comme un insecte vers la lumière ", " un second lieu de naissance " (ibid., 85). L'étude conclut, sur la base de ces données, que le konbini constitue un " espace de réconfort " hors du foyer. D'autres enquêtes nous apprennent qu'environ $35 \%$ de la génération des 20-29 ans disent s'arrêter "souvent " voire "presque tout le temps » dans des konbini sans intention d'acheter (et l'on obtient un chiffre de $70 \%$ en ajoutant ceux qui s'y arrêtent "de temps à autre »). En conséquence, il apparaît que ces jeunes ne "peuvent imaginer leur quotidien sans konbini » et qu'ils trouvent « amusant de visiter ce lieu pour lui-même » (Oeda, Ebisawa, Shibuya, Hashimoto \& Hayashi 2004 : 231).

En résumé, on peut voir qu'au tournant des années 2000, les adolescents et vingtenaires ont commencé à fréquenter les konbini non pas uniquement pour leurs achats, mais aussi comme lieu de rendez-vous, pour s’y délasser ou feuilleter des revues. Libérés de relations interpersonnelles subies, ils s'y réservent un "territoire à eux " où ils peuvent s'immerger dans un univers rempli de magazines et de biens de consommation inédits. Les konbini autorisent une existence solitaire, assurent un confort et de multiples sources de satisfaction, à l'écart de la famille et du quartier. C'est pourquoi la communication entre client et vendeur d'une part, entre les clients d'autre part, s'établit en interagissant le moins possible, dans un souci de préserver l'intimité de chacun. L'espace des konbini, plutôt que d'encourager les échanges entre soi et les autres, créerait au contraire d'ingénieuses séparations, maintenant ainsi des lignes de démarcation individuelles. Fujimoto (2013) a même remarqué qu'une communication oblique se mettait en place dans les konbini, "où des égaux en timidité peuvent se réunir ».

De plus, les utilisateurs des konbini, depuis les années 2000, ne se composent plus seulement de jeunes adultes. Alors que durant les années 1990, Seven-Eleven comptait à peine $9 \%$ de clients de plus de 50 ans, leur proportion a atteint $20 \%$ dès 2007 (Kawabe 2007). Les konbini ont effectivement développé des stratégies commerciales destinées à une clientèle d'âge moyen et avancé. Selon une enquête d'Oeda et al., bien que la génération des 50-60 ans se plaigne que les konbini participent à la désertification des rues, nuisent à la qualité des paysages urbains, vendent de la nourriture sous vide, parce qu'elle y trouve ce qui lui est essentiel, elle constitue « de tous les groupes interrogés, celui auquel la proximité d'un konbini procure 
le plus fort sentiment de sécurité " (Oeda, Ebisawa, Shibuya, Hashimoto \& Hayashi 2004 : 231). Depuis 2001, Seven-Eleven conduit à Tama New Town des « entretiens en porte-à-porte " visant les personnes âgées vivant seules, toujours plus nombreuses. Depuis 2004, tous les magasins SevenEleven sont incités à mener ces entretiens personnalisés (Shükan tōyō keizai 週刊東洋経済 ${ }^{9}, 6$ juin 2007).

$\mathrm{Si}$ le konbini, en tant qu'espace de consommation, incarne aux yeux des jeunes un lieu émancipateur [...], les seniors y voient au contraire un lieu protecteur, qui leur permet de demeurer autonomes, même lorsque parents et proches commencent à manquer. Sans se substituer à la sphère familiale et communautaire, les réseaux de konbini se sont toutefois mués en une infrastructure indispensable, qui soutient la consommation quotidienne des individus de toutes les générations. Soulignons que lors du séisme majeur qui a frappé le Nord-Est du Japon en 2011, les konbini ont été utilisés comme interfaces d'un réseau d'entraide et de secours aux victimes (Kawabe 2011). On raconte encore que dans les régions dévastées par les tremblements de terre de Hanshin-Awaji et de Niigata Chūetsu ${ }^{10}$, ils ont fourni des plateaux-repas, des sōzai 惣菜 (denrées alimentaires) et de l'eau, ce qui leur a valu le surnom de «variante privée de la ligne d'approvisionnement de secours" (minkanban raifurain 民間版ライフライン).

Enfin, l'administration des CVS se caractérise par des procédures de recrutement ainsi que des techniques de contrôle opérationnel dignes d'une grande entreprise, ce qui les distingue de la gestion traditionnelle des commerces alimentaires, familiale et/ou endogame. Le konbini intègre dans une surface de vente standardisée de nombreux magasins spécialisés autrefois indépendants, souvent gérés en auto-entrepreneuriat (primeurs, marchands de spiritueux ou de riz). Dès lors, même si l'assortiment des articles et l'agencement des surfaces peuvent s'adapter aux singularités logistiques et aux besoins locaux, de nombreux éléments (la nourriture sous vide, les services bancaires et de distribution d'argent liquide, les journaux et magazines, etc.) sont uniformisés au niveau national.

9. Magazine hebdomadaire consacré à l'actualité financière et économique. (N.D.T.) 10. Le grand tremblement de terre de Hanshin-Awaji a notamment touché l'agglomération de Kōbe le 17 janvier 1995. Celui de Niigata s'est produit le 23 octobre 2004. (N.D.T.) 
Au moment de leur introduction, les premiers CVS passaient pour des magasins où les relations de vendeur à client étaient plus personnalisées, ce qui les rendait plus conviviales que celles permises par les supermarchés ou les centres commerciaux. On supposait alors qu'une communication de proximité serait assurée par des commerçants possédant leur magasin et y employant leurs parents - les konbini fonctionnant sur le principe de la franchise. Il faut se rappeler que dans les années 1970, plus de 730000 épiceries étaient en activité, dont 85 \% en gestion familiale (Kawabe 2006). À partir des années 1980, on observe une augmentation du nombre de konbini dont la gestion est confiée à des familles, mais pas seulement : un management axé sur des relations contractuelles (salariés à temps complet ou partiel, employés en contrat court ou arubaito アルバイト) se développe, afin de faciliter les roulements de personnel. Et même si des détaillants autrefois propriétaires d'épiceries indépendantes ont pu ouvrir leur magasin franchisé en réorganisant leurs méthodes de travail, des salariés ayant quitté leur entreprise ${ }^{11}$ aussi bien que des personnes morales (sociétés de grande distribution, chaînes d'hôtels, entreprises) se sont imposés en tant qu'acteurs-clés du marché des konbini. En 2000, environ 50 \% des propriétaires de konbini ayant signé un contrat de franchisage avec une marque, étaient auparavant salariés d'une entreprise, contre à peine $14 \%$ des gérants de " magasins de vins et spiritueux » indépendants (Kawabe 2007). Alors qu'il était courant jusqu'aux années 1980 de voir se former de petites chaînes d'épiceries à l'échelle d'une région, en particulier grâce à des associations volontaires de détaillants, elles ont été peu à peu absorbées par des sociétés à forte capitalisation. Pour ces raisons, les CVS qui se situaient dans la lignée des petits commerces bien ancrés territorialement, voient se diluer leur semblant de localisme et se convertissent en points d'amarrage de travailleurs contractuels, remplaçables. [...]

On peut ainsi dire que le réseau des konbini permet aux individus d'échapper aux relations familiales ou sociales traditionnelles et locales, tout en leur rendant service à deux titres, comme travailleurs ou producteurs et comme consommateurs. Les konbini ont procuré à beaucoup de gens la jouissance d'être «des individus ». Par exemple, le journal Nikkan Sports a

11. En japonais, datsusara 脱サラ, un salarié qui a démissionné de sa compagnie pour créer son entreprise. (N.D.T.) 
fait paraître, du 16 janvier au 15 février 1997, une série d'articles intitulée "J'adore les konbini » (vingt-six au total). Le premier portrait présenté était celui d'un jeune homme ayant remporté le championnat des amateurs de konbini, lors de l'émission "TV Champion " diffusée par la chaîne TV Tokyo (Terebi Tōkyō テレビ東京). En 1986, il avait quitté sa ville d'Amagasaki (dans le département de Niigata) afin de poursuivre ses études dans la capitale, où il commença à vivre seul et découvrit les konbini. « Je me régale du simple fait de regarder tous ces objets chargés de couleurs, comme les paquets de chips. J'attends avec impatience la sortie de nouveaux produits, et il m'est arrivé de rester jusqu'à 18 heures par jour dans une boutique ". Il est par la suite devenu consultant en konbini, qu'il qualifie d'espaces fantasmagoriques. Et il n'est pas le seul, bien qu'il s'agisse d'un cas extrême : les discours témoignant d'une passion pour les konbini sont légion. [...]

On mentionnera enfin les étudiants qui se rendent dans un magasin tard dans la nuit et y lisent debout pendant près de cinquante minutes, au motif que «la solitude se dissipe mieux quand on passe du temps dans un magasin lumineux, plutôt que seul chez soi dans le noir" (Asahi shinbun 朝日新聞, 17 février 1997). Parfois appelés « électro-aimants des konbini ", les journaux et revues servent en effet autant de produits disponibles à la vente que de dispositifs pour attirer les potentiels clients. La perception des "clients-lecteurs " est en outre positive : ils accroissent le sentiment de sécurité des autres usagers (Yamada 1996 : 37). Et pendant qu'on observe en coin les lecteurs visibles depuis la vitrine translucide ou les clients qui déambulent d'un rayon à l'autre, on cède pareillement à l'envie d'examiner les denrées ou de feuilleter à son tour. On n'a qu'à baisser les yeux sur les revues et les articles, et il n'y a alors pas besoin de croiser le regard des autres, ni d'engager la conversation avec eux. Tous ces individus se contentent de partager un espace-temps bref et étroit. Ceux qui vont au konbini savent ainsi comme il est plaisant de se mêler à un groupe d'inconnus qu'on aura oubliés l'instant d'après. [...] 


\section{Espace-temps et mise en réseau dans les convenience stores}

Comme nous l'avons expliqué, les CVS se présentent comme un maillage dense d'échoppes couvrant l'ensemble du territoire national, étroitement liées par un système de franchises et dont l'efficacité se fonde sur des techniques logistiques et numériques sophistiquées. En bref, l'une de leurs réalisations majeures réside dans la construction d'un horizon d'attente, en vertu duquel des magasins sont à disposition n'importe quand, n'importe où, soit un réseau ubiquitaire dans l'espace et le temps (Tanaka 2006). En particulier, les konbini ne commercialisent pas seulement des produits vendus à l'unité, mais bel et bien l'idée de leur commodité, permise par leur réticularité. Par exemple, les produits y sont rarement soldés, de sorte qu'ils coûtent relativement cher par rapport à ceux d'autres enseignes. On recourt malgré tout aux konbini, parce qu'ils donnent aisément accès à des biens et services de proximité partout, à tout moment : ils ont un caractère intrinsèquement pratique. La valeur marchande attribuée à leur confort d'usage dépend précisément de leur mise en réseau, et les consommateurs, qui paient le prix de ce confort, réalisent ainsi une consommation en réseau.

À l'origine, les premiers konbini constituaient un format de magasin autorisé à s'implanter dans les rues marchandes, là où les grandes surfaces n'avaient pas le droit de s'installer, suivant les règles de l'ancienne loi sur les Commerces de détail de grande envergure (Daikibo kouri tenpo hō 大規 模小売店舗法), datant de 1973. Autrement dit, les premiers CVS venaient en complément des commerces indépendants de taille petite et moyenne ainsi que des supermarchés ou galeries marchandes, comblant les disparités d'offre commerciale dans les aires urbaines. Mais au fur et à mesure que ces espaces de consommation auxiliaires devenaient un large circuit de mieux en mieux organisé, ils ont développé un système s'emparant des autres types de commerces de détail, et contrôlant les étapes de la production, de la consommation et de la distribution. [...]

Au-delà de leur rôle d'espace de consommation local, leur appartenance à un réseau aux dimensions régionales est reconnue. Cela signifie que la consommation n'est qu'une partie de leurs fonctions : les konbini peuvent en incorporer bien d'autres via leur insertion dans un réseau, dont ils deviennent un nœud. On citera comme exemples de rôles que les konbini ont peu à peu endossés : l'information (journaux, magazines, etc.), la logistique (services de poste et de livraison), les services financiers (possibilité d'y souscrire à une police d'assurance, d'y retirer de l'argent, d'y régler ses 
factures), le travail (du fait de leur participation à un marché du travail flexible), la gestion des risques (avec leur mutation en zones d'assistance et de protection en cas de catastrophe), la gouvernance (pour les procédures nécessaires entre autres à l'obtention d'un certificat de résidence). En intégrant des fonctions multiples qui renforcent leur nature réticulaire, les konbini s'avèrent aussi compatibles avec les logiques du néolibéralisme, qui pousse à la déréglementation.

Les konbini vont d'ailleurs contribuer à flexibiliser l'expérience de l'espace et du temps. Selon les usagers qui se sont exprimés dans le cadre d'une enquête sur les « lieux où l'on pénètre naturellement parce qu'on les remarque en marchant » (Kurihara \& Tomishima 1998 : 85), par exemple, les konbini incarnent des flux de vie et de mouvement, qu'ils soient habituels ou inhabituels - toujours, d'emblée, présents "au milieu de l'action " - et l'on s'attend à ce qu'ils entrent régulièrement dans notre champ de vision. Il ne s'agit pas de monuments irremplaçables et fixes que l'on se doit de visiter, même s'ils nécessitent un déplacement inhabituel; ce sont plutôt des espaces [...] imbriqués dans les déplacements des populations.

Cette imbrication des konbini dans les migrations et les mouvements humains est sous-tendue par de vastes opérations statistiques et d'ingénierie [...]. Selon un ancien responsable des relations publiques chez SevenEleven, « l'implantation d'un nouveau centre commercial ou supermarché engendre des passages de visiteurs et de voitures, dont il n'est pas question avec les konbini. Ils ne peuvent émerger que là où s'agglomèrent déjà des flux humains et automobiles"(Forbes, novembre 2002: 72). À la différence des sites touristiques qui suscitent des flux de visiteurs venus pour le spectacle offert, les konbini émergent de la concentration de trajets multiples. Les horaires d'ouverture et de fermeture sont donc planifiés en correspondance avec les mobilités des passants et des habitants du quartier. À cette fin, l'exploitation de techniques démographiques pour analyser les flux de véhicules et de piétons, et d'outils de géographie économique pour visualiser les aires de chalandise dans les régions visées, permet d'identifier les zones les plus propices à l'ouverture d'un point de vente. Dans les banlieues, par exemple, l'entrée à la gauche des piétons juste après avoir traversé un carrefour est jugée plus aisée ${ }^{12}$, en raison des feux rouges qui

12. Le Japon faisant partie des pays où l'on conduit à gauche. (N.D.T.) 
ralentissent la vitesse des piétons et des automobilistes. Dans le cas des rues commerçantes à la sortie des gares, la partie voisine des zones résidentielles est préférée. En outre, la disposition des parkings fait appel à l'ingénierie des transports et la présentation des produits s'inspire de recherches en psychologie. Les sciences de l'information vont également contribuer au développement de nouveaux produits et services (Forbes 2002 : 72). Dès 2001, Family Mart a introduit un système d'information géographique (appelé FM-GIS) permettant aux équipes de prospective de cartographier rapidement des données de trafic (ou sur l'état des réseaux) et des résultats issus des recensements nationaux [...] (Keizai 経済 ${ }^{13}$, juillet 2001).

Bien sûr, tous ces calculs et ces opérations ne sont pas infaillibles. Désormais, on ne peut s'empêcher d'espérer que partout et à n'importe quelle heure, l'on tombera sur des magasins fraîchement approvisionnés. Mais il arrive qu'on ne trouve pas de konbini au moment précis où il le faut, ou qu'on soit à l'inverse incommodé si l'on vit à côté de l'un d'eux. Premièrement, la répartition des konbini sur le territoire japonais n'est pas équilibrée : le ratio population/surface disponible diffère légèrement d'un département à l'autre ${ }^{14}$. Ensuite, leurs services ne sont pas partout identiques: certains magasins vendent des aliments frais ou des produits de terroir sans relation avec les marques des franchises auxquelles ils sont affiliés. En d'autres termes, les cas de commerces n'épousant pas parfaitement le format du CVS, mais nommés et perçus comme tels, existent encore. En fonction des régions d'ailleurs, ils étaient jadis appelés soit "convenience stores " soit "konbini ». L'abréviation konbini s'est toutefois imposée à partir des années 1990, en même temps qu'une hausse très forte du nombre d'enseignes. Cette évolution traduit une conscience de leurs similitudes, en dépit des décalages entre territoires et entre compagnies. Cela renvoie aussi à l'élimination des chaînes petites et moyennes et à la métamorphose des grandes marques en chaînes nationales, qui standardisent le secteur. Ce

13. Un mensuel d'analyse de l'actualité économique publié par Shin Nihon shuppansha 新日本出版社. (N.D.T.)

14. Par exemple, Kōchi est le troisième département le moins peuplé du Japon en 2015, et celui qui recense le plus faible nombre de konbini sur son territoire, avec 177 établissements affiliés à 15 marques en 2006; aucune borne de retrait d'argent liquide n'y a été installée avant 2006. Et, dans 13 départements, les Seven-Eleven avaient des horaires différents du reste du pays. 
raccourci montre selon nous que les konbini [...] ont réussi à dessiner un horizon d'attente où " un konbini qui en serait vraiment un se doit d'être ubiquitaire » (Tanaka 2006).

Depuis les années 1980, l'augmentation des enseignes s'est accompagnée d'une très forte croissance du nombre de magasins franchisés (fig. 1). Le nombre de chaînes a atteint son apogée dans les années 1990 (une soixantaine environ) et les établissements sont passés d'à peine 6000 unités au début des années 1980, à plus de 40000 la décennie suivante.

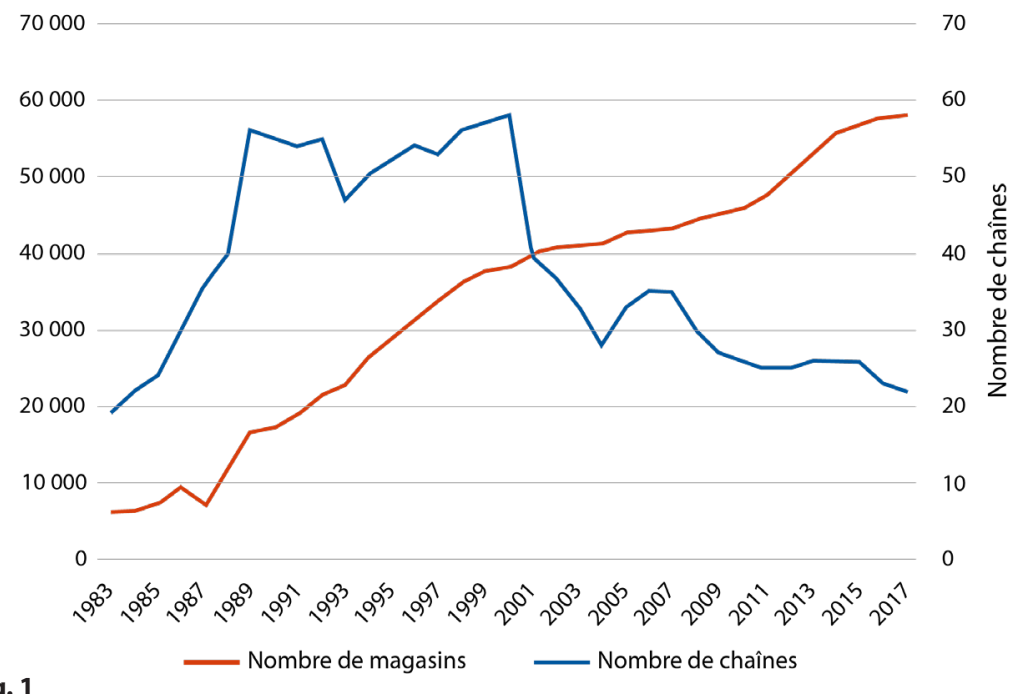

Fig. 1

Évolution nationale du nombre de convenience stores et du nombre de chaînes, 1980-2017. Source : Tanaka Daisuke 2019, d'après des statistiques issues d'une enquête menée par l'Association japonaise des franchises (Nihon furanchaizu chēn kyōkai 日本フランチャイズチェーン協会).

Cependant, sous l'effet des campagnes agressives menées par les plus grandes marques, le marché s'est nettement concentré depuis les années 2000. Si environ 2000 magasins faisaient faillite chaque année, 3000 magasins étaient inaugurés sur ce secteur très concurrentiel. Le nombre de chaînes a commencé à stagner puis le taux de croissance annuel du nombre d'établissements s'est à son tour stabilisé, aux alentours de 1000 ouvertures par an. Les compagnies ont une à une abandonné leurs projets d'expansion tous azimuts caractéristiques des années 1980 et se sont 
tournées vers des stratégies d'implantation plus sélectives, privilégiant les emplacements attractifs et condamnant sans hésiter les officines peu rentables. À partir des années 2010 cependant, l'oligopolisation du secteur stimule à nouveau les ouvertures de boutiques. En 2018, les compagnies Seven-Eleven, Family Mart et Lawson possèdent environ $85 \%$ du nombre total d'établissements franchisés en activité au Japon. On peut ainsi avancer que ce type de commerce s'est fondamentalement uniformisé.

Avec le renforcement de la concurrence, les calculs statistiques et ingénieuriaux effectués par les compagnies et décrits plus haut se sont raffinés, tout comme la vision d'une méta-organisation en réseau des konbini. C'est leur cadence d'ouverture, de clôture et de relocalisation qui fait aussi surgir cet espace-temps flexible, où les mobilités humaines orientent les apparitions et disparitions des points de vente. Le consommateur qui anticipe de faire un détour par un konbini au cours de ses trajets, ne se soucie pas de la faillite d'un magasin non rentable, à l'exception d'une poignée d'usagers invétérés. Les ouvertures et fermetures sont ainsi rythmées par le flux des corps, si bien que la spatialité même des konbini devient flottante. [...]

Qui plus est, les livraisons trois fois par jour, 365 jours par an, permettent des pratiques de consommation atemporelles, dans la mesure où il est possible de satisfaire tout besoin au moment qui nous convient, à la moindre envie, en pleine nuit ou au petit matin. Comme nous le verrons, cela reflète également un processus d'adaptation des modes de vie des travailleurs à une temporalité indéfinie, à savoir des journées de travail longues voire surchargées, ou avec des horaires atypiques. En adhérant à cet espacetemps réticulaire et manipulé, des individus dépourvus de liens avec des groupes intermédiaires (tels que la localité ou la famille) peuvent subsister. Inversement, la mesure et le contrôle des mouvements et des désirs de multiples individus, socialement désaffiliés, favorise l'émergence d'un espacetemps fluctuant et atemporel. Ces modalités permettent ainsi aux réseaux de konbini de connecter les individus.

Il faut souligner que cette entreprise de connexion, via les konbini, des désirs d'individus anonymes à un réseau constitué en méta-organisation, est propre à la société japonaise contemporaine. C'est pourquoi, dans la section suivante, nous nous appuyons sur la comparaison entre les konbini japonais et les convenience stores états-uniens pour analyser le fonctionnement spécifique des premiers. 


\section{Les konbini, un réseau japonais}

\section{L'internalisation d'un « monde extérieur » d'origine américaine : CVS et konbini, de l'importation à la ré-importation}

On date de 1927 l'apparition du premier CVS, dans l'État du Texas (Kawabe 2005). Les fondateurs de Southland Ice Corporation, ancêtre de Seven-Eleven USA, ont pressenti que la population locale voulait consommer autre chose que de la glace le dimanche et en soirée. Le premier CVS était un magasin de type drive-in à la devanture ouverte, agrémenté d'un parking et d'un comptoir en libre-service, permettant aux clients de passer commande auprès du vendeur tout en étant garés face au magasin, puis de recevoir leur achat tel qu'il était présenté sur des menus ou affiches (Kawabe 2006).

Les CVS doivent leur essor au vote puis à la promulgation de la loi sur les Autoroutes (Federal Aid Highway Act) entre 1956 et 1957 et, subséquemment, à l'expansion des réseaux autoroutiers, qui favorisent les trajets automobiles et stimulent la périurbanisation des villes à partir des années 1960 . Le nombre d'établissements, de 500 en 1957, passe à 2500 en 1960, 5900 en 1965, 13250 en 1970, puis 25000 en $1975^{15}$ (Kawabe 2006). Depuis cette époque, la plupart des CVS vendent aussi du carburant en libre-service $^{16}$. De fait, la majorité des CVS aux États-Unis servent simultanément de point de ravitaillement en essence sur le bord de la route, de sorte qu'il est matériellement impossible de les distinguer des stations-service. La prolifération des CVS, par leur parasitage de la société automobile, pose

15. Un autre facteur de cet essor est, à partir des années 1960 - et en complément du mode de gestion directe jusque-là dominant-, l'introduction d'un système de franchises, grâce auquel un propriétaire peut prendre en charge le capital initial d'un magasin, ce qui accélère les créations d'établissements. La National Association of Convenience Stores (NACS) met cela en place dès 1961.

16. La vente d'essence croît à partir des années 1970 en particulier. Le nombre de magasins Seven-Eleven dotés de stations d'essence, de 721 en 1975, s'élève à 2247 en 1980. Cela représente respectivement 23 puis $30 \%$ du total du chiffre d'affaires réalisé par ces CVS (Kawabe 2005). En 1982, environ $60 \%$ des CVS proposent du carburant, et $12 \%$ de l'essence vendue aux États-Unis provient d'un CVS en 1984. À partir de 1989, le chiffre d'affaires engrangé par la vente d'essence dans les CVS dépasse celui des achats en magasin (ibid., 90). Au cours des années 1980, entre 3000 et 4000 CVS apparaissent chaque année, mais la moitié d'entre eux naissent de la conversion d'une station d'essence (Kawabe 2003 : 10). 
des problèmes logistiques et sociaux inhérents à cette dernière, comme la congestion routière et la pollution atmosphérique. Ces problèmes ont été partiellement atténués par la mise en place de centres de distribution, visant à rationaliser les livraisons. Cependant, même les fast-foods n'étaient livrés que deux à trois fois par semaine. Cela reste assez sommaire, si l'on songe que le rythme de livraison était de trois fois par jour au Japon dès la seconde moitié des années 1980 .

En 2005, le nombre total de CVS au Japon approchait les 44000 unités (plus de 50000 en incluant les enseignes qui n'ont pas adhéré à l'Association des CVS), contre environ 140000 aux États-Unis. Sans tenir compte des caractéristiques géographiques (comme le relief), des conditions de trafic et des structures supportant la vie quotidienne, le Japon compte un konbini tous les sept kilomètres carrés, cette moyenne étant de 1 pour 67 kilomètres aux États-Unis. On peut aussi calculer que le Japon possède un magasin pour 2600 habitants à peu près, alors qu'on trouve un rapport de $1 / 2000$ dans le cas nord-américain. C'est cette forte densité de population associée à un maillage dense de magasins qui assure le roulement rapide des produits au Japon. Comme nous l'avons expliqué, les CVS aux États-Unis se sont diffusés dans le sillage des stations d'essence, dont la propagation était soutenue par la motorisation et l'étalement urbain. Par contraste, les konbini japonais sont perçus comme des « lieux que l'on remarque en marchant " (Kurihara \& Tomishima 1998 : 85) : toute leur singularité se situe là.

En ce qui concerne l'origine de l'importation des CVS des États-Unis vers le Japon, deux théories coexistent : l'une selon laquelle cette forme commerciale est introduite en 1967-1968 par une association de grossistes et de petits commerçants, l'autre la datant de 1974, quand le grand groupe Itō Yōkadō イトーヨーカ堂 ouvre le premier Seven-Eleven dans la ville de Toyosu 豊洲. La date d'importation est donc fixée en fonction de la définition donnée d'un convenience store ${ }^{17}$. Par la suite, la diffusion du système

17. Les CVS ont été initialement importés avec l'intention de créer un "style japonais de convenience store" se focalisant sur les produits frais, et veillant " aux tendances de consommation, aux traditions alimentaires et aux pratiques d'achat des Japonais" (Kawabe 2006). Kawabe note que l'on "peut appeler modèle japonais du konbini, un système de gestion avancé qui perfectionne les fonctions de magasinage, 
de la franchise dans les années 1980 et de celui des points de vente dans les années 1990, ont permis aux konbini de se transformer en réseaux denses, hautement informatisés. On peut dire à cet égard que la distinction entre convenience stores états-uniens et konbini (re)naît au moment où la possibilité d'acheter des nouveautés, partout et tout le temps, devient l'horizon d'attente ordinaire des consommateurs (Tanaka 2006). Kawabe Nobuo analyse ainsi la différence entre konbini japonais et convenience stores américains : les premiers, "en développant et perfectionnant des systèmes de gestion de l'information, afin d'harmoniser le mieux possible l'offre et la demande, ont évolué en une industrie numérique, bien au-delà de la vente au détail classique " (Kawabe 2006). Avec les konbini, la relation cyclique entre offre et demande de biens et services fonctionne en réseau, grâce à un système enregistrant à haute fréquence les réactions des clients (high-speed feedback system) [...].

Lorsque les konbini commencent à se développer de manière autonome et enregistrent des progrès très rapides, des années 1980 à 1990, les CVS états-uniens entrent au contraire dans une période de crise $^{18}$. Certains avancent même que "l'ère des convenience stores est achevée " (Kawabe 2006/8 : 92). Ce type de commerce de proximité souffre de la guerre des prix menée par d'autres formes de distribution, tandis que sa présence adjacente aux stations d'essence se réduit elle aussi. Alors que l'industrie entière était à la peine, le leader de ce marché aux États-Unis, Southland Corporation, a demandé de l'assistance pour sa rénovation au groupe Itō Yōkadō, fondateur de Seven-Eleven au Japon. En 1989, la division hawaïenne de Southland a été transférée vers Seven-Eleven Japan. Il

d'approvisionnement, de logistique et de contrôle, et les intègre dans une seule surface commerciale " (Kawabe 2006).

18. Au début des années 1990, les trois principales chainnes nationales, dont Southland Corporation (à la tête des Seven-Eleven) et Circle K, font faillite, et bien d'autres compagnies connaissent des difficultés similaires. Parmi les causes de ce marasme, on retient : 1) une hausse des taux d'intérêt des dettes engendrées par une diversification des activités et des fusions-acquisitions préventives; 2) une exacerbation de la compétition entre CVS de plus en plus nombreux, centres commerciaux périurbains et grands magasins discount; 3) une déstabilisation de la gestion des CVS, provoquée par l'augmentation des points de vente d'essence et par les investissements dans la production de carburant. 
importait alors de prévenir le risque que de " nombreux touristes japonais se rendant à Hawaï et y voyant les Seven-Eleven en activité sur cette île ne conçoivent une image globalement négative des Seven-Eleven nippons, qui nuirait à ces derniers" (Kawabe 2003 : 13). Cet objectif est demeuré, même après qu'Itō Yōkadō fut devenu l'actionnaire principal de Seven Eleven USA, au terme d'opérations de fusion-acquisition menées entre 1990 et $1992^{19}$. En conséquence, l'enseigne japonaise est devenue propriétaire de son homologue états-unien, menant concrètement à une « konbinisation » des $\mathrm{CVS}^{20}$.

Pour récapituler, le CVS a été introduit au Japon depuis les États-Unis, dans une optique de modernisation du secteur de la vente au détail. Mais en raison des trajectoires divergentes que les CVS ont suivies dans ces deux pays, lorsque Family Mart exporte ses boutiques vers les États-Unis, la forme nord-américaine des CVS lui paraît désormais frustre, de sorte que sa politique d'expansion mise sur l'image " proprement japonaise " des konbini [...]. Par contraste avec Seven-Eleven Japan, qui s'est lancé dans la rénovation de l'enseigne Seven-Eleven USA afin qu'elle gagne une image de qualité japonaise associée aux konbini, Family Mart vend aux États-Unis un modèle de magasin made in Japan, qui se veut sans rapport avec les CVS américains. Cette entreprise a conduit une enquête révélant que les habitants des États-Unis avaient, plus que les Japonais, une opinion négative des convenience stores. De nombreux CVS existent toujours en appoint des stations-services, et leurs responsables n'y voient qu'une activité secondaire. Il en résulte que le magasin paraît souvent sombre, sale, avec un service de qualité médiocre; et les femmes sont réticentes à y entrer (Nikkei Business, 4 septembre 2006 : 38-43) [...]. Donc, si Seven-Eleven a cherché à réhabiliter Southland Corporation afin de protéger son image de qualité japonaise, Family Mart s'est tourné vers une stratégie de différenciation entre

19. En 2005, afin de résoudre définitivement le décalage entre l'administration de la licence et le statut de licencié, Seven-Eleven Japan a racheté Seven-Eleven USA en en faisant une filiale à $100 \%$.

20. Il a été notamment exigé des CVS américains qu’ils modernisent leur offre de produits, leur logistique et leurs politiques de prix, avant d'introduire un système solide de points de vente. Il s'agit en définitive d'une stratégie d'enrichissement des CVS américains, destinée à les transformer en réseaux agrégeant de multiples fonctions, afin qu'ils se distinguent des stations-services et autres détaillants. 
le CVS américain et les konbini japonais, équivalents à des CVS de luxe. On aboutit alors à un curieux paradoxe, qui consiste à valoriser un produit originellement nord-américain en mettant en exergue sa japonité et, plus encore, à entretenir cette image exotique, répandue outre-Atlantique [...].

\section{Aux marges du système sous-tendant les réseaux commerciaux japonais. Le recrutement de travailleurs précaires dans les konbini}

Les facteurs qui ont permis aux konbini de former de puissants réseaux ne sont pas uniquement issus du centre de l'économie-monde que sont les États-Unis. Il faut aussi questionner les structures qui, en employant des travailleurs précaires à bas coût et interchangeables, font vivre une partie de la société japonaise et la reproduisent en tant que marge.

Un premier point concerne l'adaptation des corps des travailleurs à des rythmes de travail de 24 heures par jour, 365 jours par an, et ponctués de livraisons trois fois par jour. L'enjeu est, autrement dit, de se demander comment sont formés et recrutés des travailleurs physiquement et cognitivement adaptables à l'espace des flux et à la temporalité atemporelle conceptualisés par Castells. Jusqu'à une période récente, les horaires d'ouverture tardive des konbini reposaient sur une gestion familiale, dont les membres tenaient le magasin au détriment de leur vie privée, et sur de jeunes employés ayant le droit de travailler de nuit. Depuis les années 2000 néanmoins, ceux que l'on considère comme des employés à temps partiel peu onéreux, capables d'effectuer diverses tâches peu qualifiées, sont les actifs étrangers, les actifs âgés et les femmes au foyer. [...] Avec la parution en 2017 de l'essai de Serizawa Kensuke, Konbini gaikokujin コンビニ外国人 (Étrangers des konbini), la hausse du nombre de travailleurs migrants employés à temps partiel est devenue un important sujet de débat public. Pourtant dès les années 2000, des journaux publiaient des dossiers spéciaux prévoyant «[...] la fin des travailleurs étrangers "jetables". Si le Japon a pu jusqu'ici ne pas être dépendant des migrations de travail, cette époque est révolue " (Nikkei Business, 11 septembre 2006 : 36-39); et l'on disait déjà que "ni les konbini ni les izakaya ne [pouvaient] faire leurs affaires sans l'embauche d'étudiants étrangers" .

Selon des statistiques du ministère de la Justice (Hōmushō 法務省, MOJ [Ministry of Justice]), environ 110000 étrangers vivant au Japon en 
2006 étaient détenteurs du " permis d'exercer des activités non-qualifiées " (shikakugai katsudō no kyoka 資格外活動の許可), leur donnant le droit d'exercer un emploi à temps partiel. Dans ces circonstances, une chaîne de distribution nationale en est venue à déclarer que "l'embauche de salariés à temps partiel est à la discrétion des propriétaires de magasins. Le siège de l'entreprise n'en sait rien" (Nikkei Business, 11 septembre 2006 : 36) : on n'a donc plus une idée exacte du nombre de résidents étrangers employés.

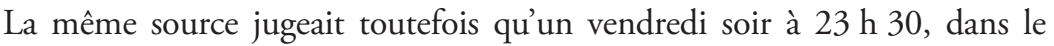
quartier de Kabukichō à Tokyo, vingt des quarante-cinq employés travaillant dans vingt-et-un konbini observés n'étaient pas Japonais.

Par ailleurs, le nombre total de travailleurs migrants serait passé de 480000 en 2008 à 1,28 million en 2017 , soit une multiplication par 2,5 (source : Cabinet du Premier ministre, chiffres sur les travailleurs étrangers publiés le 20 février 2018 ${ }^{21}$ ). Puis un amendement à la loi de Contrôle de l'immigration (shutsunyükoku kanri hō 出入国管理法), qui incorpore un nouveau statut de résident "à compétences spécifiques " (tokutei ginō 特定 技能), a été soumis lors d'une séance extraordinaire du Parlement en 2018 afin d'assouplir l'admission de travailleurs migrants. L'Association japonaise des franchises, à laquelle souscrivent toutes les grandes marques de distribution, a réagi à cette situation en publiant ses propres statistiques sur les salariés étrangers (tableau 1). Les étrangers peuvent difficilement obtenir un visa professionnel au motif qu'ils sont employés dans un konbini, car cela ne nécessite pas a priori des qualifications particulières. En conséquence, la majorité des salariés étrangers dans les konbini sont des étudiants possédant un permis de travail à temps partiel limité à 28 heures hebdomadaires. Cependant, dans le but de compenser les effets du vieillissement et du déclin de la population japonaise sur la main-d'œuvre, l'association des franchisés s'est mise à coopérer avec le gouvernement pour défendre le classement du travail en konbini parmi les emplois à " compétences spécifiques ", de manière à y stabiliser le recrutement des migrants. La publication de ces chiffres vise à faire admettre que l'activité des konbini dépend de la main-d'œuvre étrangère.

21. https://www5.cao.go.jp/keizai-shimon/kaigi/minutes/2018/0220/shiryo_04.pdf (dernière consultation le 18 septembre 2019). (N.D.T.) 


\section{Tableau 1}

Statistiques sur le nombre et la part de salarié.e.s d'origine étrangère dans les konbini en 2018.

\begin{tabular}{|l|l|l|l|}
\hline $\begin{array}{l}\text { Nom de la } \\
\text { chaîne }\end{array}$ & $\begin{array}{l}\text { Nombre de } \\
\text { salarié.e.s } \\
\text { au Japon }\end{array}$ & $\begin{array}{l}\text { Nombre de } \\
\text { salarié.e.s d'origine } \\
\text { étrangère }\end{array}$ & $\begin{array}{l}\text { Salarié.e.s d'origine } \\
\text { étrangère } \\
\text { (en \% du total) }\end{array}$ \\
\hline Seven Eleven & 390000 & 31000 & $7,9 \%$ \\
\hline Family Mart & 200000 & 10000 & $5 \%$ \\
\hline Lawson & 190000 & 11000 & $5,8 \%$ \\
\hline Mini-Stop & 34000 & 3300 & $9,7 \%$ \\
\hline
\end{tabular}

Source: Mainichi shimbun 毎日新聞, 15 septembre 2018.

Dans les faits, Lawson a confié en 2016 à une société, spécialisée dans la formation de ressources humaines au Vietnam, la mission d'y ouvrir un centre de recherche, tandis que Seven-Eleven Japan a signé en 2017 un mémorandum d'entente pour la collaboration internationale, incluant des conventions de stage avec les six plus grandes universités vietnamiennes. Ainsi, les grandes entreprises japonaises de la distribution ont petit à petit sécurisé des circuits d'approvisionnement en main-d'œuvre depuis l'Asie.

Cela ne vise pas que les vendeurs. De nombreux travailleurs étrangers peu rémunérés travaillent dans les usines à la préparation des plateaux-repas et des autres plats à la base de la culture du "repas en solo". Les barquettes sont confectionnées pendant la nuit, afin d'être les plus fraîches possibles lors de leur livraison. Les employeurs ont tendance à faire appel à des travailleuses d'origine sud-américaine qui acceptent ces conditions de travail car de nombreuses femmes mariées japonaises travaillant à temps partiel trouvent le travail de nuit pénible (Weekly toyo keizai, 16 septembre 2006 : 52). De plus, la rémunération des travailleurs étrangers atteignait au début des années 2000 seulement trois-quarts de ce qui est versé aux salariés japonais, et la rémunération des femmes employées dans les konbini équivalait à son tour aux trois-quarts du salaire versé aux travailleurs étrangers masculins (Watanabe 2005 : 88-89).

Le niveau de numérisation requis pour approvisionner les konbini et les ouvrir 24 heures sur 24 nécessite une organisation logistique complexe. En particulier, la livraison trois fois par jour suppose que des chauffeurs de poids lourds roulent durant de longues heures afin de naviguer entre les ateliers de 
transformation alimentaire, les centres logistiques et chaque magasin. Des cas de journées de 14 heures (commençant à 18 heures et s'achevant le matin suivant à 8 heures) ont été relatés (Takahisa 2003 : 41). Il s'agit en outre d'un système destiné à fournir les produits " juste à temps ». Il est donc inconcevable de ne pas les amener exactement à l'heure prévue, même si les conditions météorologiques ou de trafic se révèlent incertaines. Les chauffeurs sont dès lors forcés de ne jamais s'interrompre et, faute de pauses, les services de nuit et le surmenage les épuisent. "Mais ils doivent faire ce travail pour vivre " (Takahisa 2003 : 41). Dans les interstices de réseaux informatisés aux dimensions abstraites, les corps souffrent, soumis à un labeur incessant pour tenir la cadence. Les déréglementations dans le secteur du transport de fret et de passagers ont exacerbé la concurrence, au point que les accidents causés par des chauffeurs surmenés de poids-lourds ou d'autocars low-cost sont considérés aujourd'hui comme un problème de société, mais les systèmes de livraison desservant les konbini sont aussi en cause. Par ailleurs, la nourriture que peuvent ingérer en un temps limité les employés du secteur des transports, mobilisés de 14 à 17 heures par jour, se compose fréquemment de plats vendus dans les konbini (Shinbun Akahata しんぶん赤旗, quotidien du Parti communiste japonais, 25 avril 2003). [...] On se rend ainsi compte que ces chaînes commerciales sont parvenues à bâtir des cycles quotidiens de production-consommation qui marchent quasiment en espace clos. Un autre cycle implique les travailleurs à temps partiel ramenant chez eux les plateaux-repas périmés, et qui font ainsi une partie de leurs courses sur leur lieu de travail. Dans un konbini où une myriade d'individus passe dans une grande fluidité, même la distinction entre " travailleurs » et « clients " devient contingente.

À l'instar des conducteurs et des vendeurs, les gérants de magasins sont forcés de s'adapter à des journées de travail longues et surchargées. "Si un mitemps annule à la dernière minute, je me farcis 36 heures de travail à la suite ", " je ne peux jamais partir en vacances "... D'après les services responsables de la gestion des ressources humaines, chaque magasin a besoin de deux roulements (de douze heures par jour), répartis généralement entre le propriétaire et son épouse, avec un jour de repos par semaine, soit 7200 heures de travail à l'année. On dit que l'exploitation d'un konbini se fonde sur l'hypothèse que chaque salarié à temps plein peut supporter une durée de travail équivalente à plus de 1,5 fois celle d'un salarié d'entreprise conventionnelle, à savoir 2000 heures par an (d'après une dépêche de Rengō tsüshin 連合通信, Rengo News, $1^{\text {er }}$ mars 2001). Depuis longtemps médiatisés, les conflits juridiques entre 
franchiseurs et franchisés n'ont pas trouvé de solution durable, et la gestion des ressources humaines des CVS va jusqu'à être qualifiée d'esclavage (Honma 1999). On assiste plus récemment à l'émergence du problème du surmenage des couples de propriétaires et des " petits boulots au noir » (burakku baito ブ ラックバイト) qui l'accompagnent (Mitsuzono 2015).

Dans un contexte où l'individualisation des modes de vie progresse et repose sur l'existence des konbini, ces derniers poursuivront leur recrutement d'êtres humains capables de tolérer la sévérité des conditions d'emploi induites par l'espace des flux et la temporalité atemporelle, en échange d'un salaire faible. Il s'agissait au départ d'étudiants ou de jeunes actifs qui y travaillaient provisoirement; mais ce sont désormais des personnes marginalisées sur le marché du travail japonais, c'est-à-dire des travailleurs âgés, des migrants venus majoritairement d'Asie et des femmes, comme les a d'ailleurs catégorisés un dossier spécial du magazine industriel Konbini : "Mamans, seniors, étrangers" : comment les engager, les former et les garder ?" (mars 2017). [...]

Alors qu'il était réalisateur en chef du dessin animé Urusei yatsura 2: byūtifuru dorimā うる星やつら2:ビューテイフルドリーマー22, paru en 1984, Oshii Mamoru 押井守 dépeint une ville qui perd conscience du temps linéaire et "objectif» (progressant du passé vers le futur), répète un éternel présent et, faute d'issue, commence à tomber en ruines. Cette ville coincée dans un espace-temps absurde dispose malgré tout de konbini qui, pour une raison quelconque, opèrent continuellement et sous-tendent le quotidien fantasmatique des protagonistes, sans se tarir. Oshii a également représenté un konbini dans le film d'animation Ghost in the Shell 2 : Innocence, paru vingt ans plus tard. Ils sont décrits comme des lieux où les cerveaux de membres de brigades anti-émeutes cybernétiques sont vulnérables au piratage, car ils y font régulièrement un détour avant de rentrer chez eux. Chacune de ces œuvres évoque un effacement des frontières entre le rêve et la réalité, entre le virtuel et le matériel, et les konbini y font figure de lieux où cet estompement produit une torsion, comparable à un ruban de Möbius [...]. Les portes automatiques des konbini sont l'entrée d'un réseau où sont manufacturés nos désirs d'obtenir ce que l'on veut en permanence, à chaque coin de rue; elles ouvrent aussi un

22. La version française du titre est Lamu : un rêve sans fin. Dans ce film, les héros mènent une enquête pour comprendre pourquoi chaque journée semble se dérouler comme la précédente, au point que les habitants de la ville perdent la notion du temps. (N.D.T.) 
champ d'exploration des rouages qui peuvent transformer le rêve d'un capitalisme informationnalisé en cauchemar.

\section{Conclusion}

Ce texte analyse les enjeux posés par l'influence des technologies numériques et l'individualisation sociale dans le Japon contemporain, au prisme des konbini. Ceux-ci actionnent des réseaux ajustés aux individus et des individus ajustés aux réseaux, le tournant numérique du capitalisme constituant leur soubassement. Ce faisant, ils redélimitent l'étendue de la société japonaise en se référant à - et en dépendant de - deux « mondes extérieurs " : les ÉtatsUnis (" centre ») pour le mode de vie, l'Asie ("périphérie ») pour le travail précaire. On peut en effet les définir comme des réseaux connectant directement des individus à la société japonaise, en se passant des attaches familiales et communautaires [...]. En pratique, les konbini se sont transformés en "postes de sécurité ", et ces lieux attirant d'innombrables individus font désormais office de réseaux administratifs d'envergure nationale. Cependant, dans un futur pas si lointain où les travailleurs étrangers représenteront plus de $10 \%$ de la main-d'œuvre, sera-t-il encore possible de qualifier les konbini d'espaces "typiquement japonais " ? En leur qualité de circuit de distribution, même s'ils circonscrivent en quelque sorte les limites de la société, ils constituent aussi un média qui en érode le fond.

Les konbini forment à la fois un réseau homogène et un espace-temps diversifié, où se côtoient jeunes et moins jeunes, consommateurs et travailleurs, ainsi que des résidents ou visiteurs étrangers. [... Récemment, les stratégies assimilant les konbini à des lieux conviviaux ou des cafés ont été adoptées par les entreprises, afin que les clients les apprécient pour leur emplacement même. Les konbini abritent ainsi des espaces-temps qui nous interrogent sur l'émergence de relations interpersonnelles détachées des appartenances communautaires et collectives traditionnelles, et sur la forme des nouveaux espaces où ces relations se nouent ${ }^{23}$. Les konbini constituent enfin une illustration

23. Sur ce point, Miura (2013) a émis l'hypothèse que l'ultra-vieillissement de la population japonaise mènerait à la formation de "komubini ", combinant des fonctions communautaires avec celles d'un konbini. 
probante et originale du pouvoir et des limites de la globalisation économique et des sociétés de l'information: même les réseaux les plus performants ne peuvent s'abstraire de l'espace, et c'est la difficulté à considérer leur matérialité et leur inscription territoriale qui engendre des souffrances sociales.

Traduit du japonais et annoté par Sophie BUHNIK.

\section{Bibliographie}

\begin{abstract}
AsABA Michiaki 浅羽通明 1990
Nemuranai Yokubō no jūten sōchi. "Konbiniensu sutoa" no Shihonron 眠ら ない『欲望』の充填装置一〈コンビニエンス ストア〉の『資本論』一 (Des équipements pour assouvir une soif insomniaque. Le capitalisme des commerces de proximité), 別冊宝島 110:80年代の正体!

(Revue Takarajima numéro spécial 110: «La réalité des années 1980 ! »), JICC shuppankyoku JICC 出版局.
\end{abstract}

\section{CAstells Manuel 1996}

The Rise of the Network Society. The Information Age: Economy, Society and Culture, vol. 1, Oxford, Blackwell.

\section{Chūō chōsasha 中央調査社 (Institut national d'enquête et d'opinion publique) 2001 \\ « Zenkoku seijin no konbiniensu sutoa riyō jōkyō » 全国成人のコンビニエンス ストア利用状況 (Les usages des CVS au}

sein de la population japonaise adulte), Chūō chōsahō 中央調査報, 12 p. https://www.crs.or.jp/data/pdf/cvs.pdf (dernière consultation en novembre 2019).

HiRATA Eriko 平田絵理子, MatsutanI Yasuko 松谷康子 \& NAITō Michitaka 内藤 通孝 2005

« Joshidaisei ni okeru konbini riyō to seikatsu shūkan no kanren » 女子大生に おけるコンビ二利用と生活習慣の関連 (Relation entre usages du konbini et modes de vie chez les étudiantes), Sugiyama jogakuen daigaku kenkyū ronshū 椙山女学園大学研究論集 (Bulletin de recherche de l'université féminine de Sugiyama), $36:$ 93-100.

HondA Shigeki 本田重紀 2009

Konbini no hikari to kage コンビニの 光と影 (Lumières sur les konbini), Tokyo, Kadensha 花伝社.

Fuנıмото Ken.ichi 藤本憲一 2013

« Hitomishiri dōshi ga tsudou kyūsuijo 
“konbini”»人見知りどうしが集う給水所 「コンビニ」(Une source de rencontres pour les introvertis : les « konbini»), in CHIKAMORI Takaaki 近森高明 \& KUDŌ Yasunori 工藤保則 (dir.), Mujirushi toshi no shakaigaku 無印都市の社会学 (Sociologie des villes sans signes distinctifs), Kyoto, Hōritsubunka-sha 法律文化社.

\section{FUJIMURA Atsuo 藤村厚夫 1991}

«Konbiniensu-sutoa ron » コンビニエンス・ ストア論 (Essai sur les convenience stores), in Serizawa Shunsuke 芹沢俊介 (dir.), Shōhi shihonshugiron 消費資本主義論 (Théorie du capitalisme de la consommation), Tokyo, Shin.yōsha 新曜社.

\section{IGARASHI Tarō 五十嵐太郎 2006}

Utsukushii toshi, minikui toshi : gendai keikanron 美しい都市・醜い都市一現代景 観論 (Beautés et laideurs des villes. Théorie du paysage moderne), Tokyo, Chūō kōron shinsha 中央公論新社.

KaWABE Nobuo 川辺信雄 2003

«Tōsan to saiken no keieishi. Sebunirebun Inc. (kyū Sausurandosha) no jirei kenkyū » 倒産と再建の経営史一 セブンーイレブン, Inc. (旧サウスランド社)の 事例研究一 (Entre faillite et réorganisation : la trajectoire de Seven Eleven [ex-Southland Corp.]), Waseda shōgaku 早稲田商学 (Revue d'études commerciales de l'université Waseda), 397 : 1-53.

\section{KaWABE Nobuo 川辺信雄 2005-2007} «Konbini zenshi » コンビ二全史 (Histoire complète des convenience stores : série d'articles parus entre 2005 et 2007),
Gekkan konbini 月刊コンビニ (Konbini, magazine mensuel).

https://commerce.media/convenience/ (dernière consultation en novembre 2019).

KaWABE Nobuo 川辺信雄 2011

Higashi Nihon daishinsai to konbini 東日本大震災とコンビニ (Le séisme du 11 mars 2011 et le rôle des konbini), Tokyo, Waseda daigaku shuppanbu 早稲田大学出版部.

KuriHARA Yumi 栗原有美 \& Tomishima Sayaka 富嶋紗綾香 1998 «"Konbini" ni okeru gakusei no shōhi kōdō. "Konbini" wa iyashi no ba ka » 『コンビニ』における学生の消費行動一 『コンビ二』は癒しの場か一

(Comportements de consommation des étudiants dans les konbini. Les konbini sont-ils des lieux de résilience ?), Kōnan kasei, Kōnan joshi daigaku kaseigaku $k e n k y u \bar{u}-k a i$ 甲南家政、甲南女子大学家政 学研究会 (Kōnan études domestiques, bulletin de l'université féminine de Kōnan), 33 : 77-91.

Matsubara Ryūichirō 松原隆一郎 2000 Shōhishihon shugi no yukue. Konbini kara mita Nihon keizai 消費資本主義のゆくえ一 コンビニから見た日本経済一 (Le futur du capitalisme consumériste : l'économie japonaise du point de vue des convenience stores), Tokyo, Chikuma shobō 筑摩書房.

Mıtsuzono Isamu 満園勇 2015

Shōtengai wa ima hitsuyō na no ka? 商店 街はいま必要なのか (A-t-on aujourd'hui 
besoin des rues commerçantes ?), Tokyo, Kōdansha 講談社.

MIURA Atsushi 三浦展 2001

Mai hōmuresu chairudo. Imadoki no wakamono o rikai suru tame no nijūsan no shiten マイホームレスチャイルドー今 どきの若者を理解するための 23 視点一 (Les enfants sans domicile fixe. 23 perspectives pour mieux comprendre la jeunesse d'aujourd'hui), Tokyo, Kurabu hausu クラブハウス.

Miura Atsushi 三浦展 2004

Fasuto fūdoka suru Nihon. Kōgaika to sono byōri ファスト風土化する日本一 郊外化とその病理一 (La fast-füdoïsation du Japon. L'étalement urbain et ses pathologies), Tokyo, Yōsensha 洋泉社.

\section{MIURA Atsushi 三浦展 2013}

Nihonjin wa korekara nani o kau no ka? 日本人はこれから何を買うのか?

(Qu'achèteront les Japonais désormais?), Tokyo, Kōbunsha 光文社.

Mīto jānaru henshūbu ミート・ジャーナル 編集部 (Rédaction du mensuel Journal de l'alimentation) 2000

«Chūkōnen dansei no konbiniensu sutoa (CVS) riyō ni kansuru chōsa » 中高年男性のコンビニエンスストア (CVS) 利用に関する調査 (Enquête sur les usages des konbini chez les hommes d'âge moyen ou avancé), Mīto jānaru ミート. ジャーナル, 37, édition de mai 2000.

\section{MoRIWAKI Takeko 森脇丈子 2006}

«Konbini riyōgata no shōhi kōdō to nipponteki kaimono shūkan. Nihon de konbini ga hayaru riyū » コンビニ利用型の
消費行動と日本的買い物習慣一日本で コンビニが流行る理由ー (Comportements de consommation liés aux konbini et habitudes d'achat au Japon. Les facteurs de la popularité des konbini), Shōkei ronsō, Kagoshima kenritsu tanki daigaku 商経論叢、鹿児島県立短期大学 (Revue annuelle d'économie et de gestion du collège universitaire préfectoral de Kagoshima), 56: 1-25.

Murata Sayaka 村田沙耶香 2016 Konbini ningen コンビニ人間 (Konbini), Tokyo, Bungei shunjū 文藝春秋.

\section{OGATA Ayako 緒方彩子 1999}

«Chūkōnen dansei no konbiniensu sutoa (CVS) riyō ni kansuru chōsa » 中高年男性のコンビニエンスストア (CVS) 利用に関する調査 (Enquête sur I'utilisation des CVS par les hommes d'âge moyen ou avancé), LDI Report, $107: 31-49$.

Ōeda Chikako 大枝近子, Ebizawa Narumi 海老澤成亨, SHIBUYA Shōzō 渋谷昌三， HASHIMOTO Yōko 橋本洋子 \& HAYASHI Tomoko 林知子 2004 «Sedaikan ni miru konbiniensu sutoa no riyō jōkyō » 世代間に見るコンビニエンス・ ストアの利用状況 (Les pratiques du konbini selon les générations), Mejiro daigaku ningen shakaigakubu kiyō 目 白大学人間社会学部紀要 (Journal du département de sociologie humaine de l'université Mejiro), 4 : 219-232.

\section{SERIZAWA Kensuke 芹澤健介 2018} Konbinigaikokujin コンビ二外国人 (Étrangers des konbini), Tokyo, Shinchōsha 新潮社. 
TAKAGI Naoto 高木直人 2005

Konbiniensu sutoa no riyō : Chūgakusei to daigakusei コンビニエンス・ストアの 利用一中学生と大学生一 (Utilisation des convenience stores chez les collégiens et les étudiants), Kure daigaku tanki daigakubu kiyō 吳大学短期大学部紀要 (Bulletin du collège universitaire Kure), 8 (18) : 11-22.

TAKAHISA Nagamichi 高久永道 2003 «FC no hikari to kage (29): Konbini ga umu “karō torakku” » FCの光と影29 一コンビニが生む「過労トラック」(Dire la vérité sur les franchises commerciales : konbini et conducteurs surmenés), VERDAD, 9 (1) : 40-42.

TAKI Kōji 多木浩二 1994

Toshi no seijigaku 都市の政治学 (Pour une science politique de la ville), Tokyo, Iwanami shoten 岩波書店.

\section{TAmURA Kaoru 田村馨 1996}

« Daigakusei no CVS riyō no jittai to ryūtsū ni taisuru ninshiki. Ankēto chōsa no shūkei kekka »大学生のCVS利用の 実態と流通に対する認識一アンケート調査 の集計結果一 (Usage des konbini chez les étudiants et conscience du système de distribution : résultats agrégés d'enquêtes par questionnaire), Shōgaku ronsō, Fukuoka daigaku sōgō kenkyūjo 商学論叢、福岡大学総合研究所 (Journal des sciences du commerce de l'université de Fukuoka), 40 (3) : 1029-1044.

TANAKa Daisuke 田中大介 2006 « Konbini no tanjō. Senkyūhyakukyūjū nendai ni okeru shōhikūkan no CMCteki kōzō tenkan »コンビニの誕生

一1990年代における消費空間のCMC的

構造転換 (La naissance des konbini :

restructuration des espaces de consommation dans les années 1990 grâce à la communication assistée par ordinateur), Nenpō shakaigaku ronshū, Kantō shakaigakkai 年報社会学論集、関東 社会学会 (Revue annuelle de sociologie, Société de sociologie du Kantō), 19 : 201-211.

\section{TANAKA Daisuke 田中大介 2007}

"CVS no "sēfuti sutēshonka" no ronri. 2000 nendai ni okeru shōhikūkan no kanri shakaiteki henyō »CVSの 「セーフティステーション化」の論理 -2000年代における消費空間の管理社会的 変容一 (Logiques de mutation des CVS en "safety stations" : évolution sociale du contrôle des espaces de consommation dans les années 2000), Shakaigaku jānaru, Tsukuba daigaku shakaigaku kenkyūshitsu 社会学ジャーナル、筑波 大学社会学研究室 (Journal de sociologie, université de Tsukuba), 32 : 61-76.

\section{TANAKA Daisuke 田中大介 2008}

«Metashikaku to shite no nettowāku :

M. Kasuteru no nettowāku gainen no kanōsei » メ夕視角としてのネットワーク 一 $\mathrm{M}$ •カステルのネットワーク概念の可能性 (Le réseau comme méta-perspective : potentialités du concept de réseau théorisé par M. Castells), Nenpō shakaigaku ronshū, Kantō shakai gakkai 年報社会学論集、関東社会学会 (Revue annuelle de sociologie, Société de sociologie du Kantō), 21 : 13-24. 
TANAKa Daisuke 田中大介 2010

«Konbini no bunka » コンビニの文化

(Culture du konbini), in InOue Shun

井上俊 \& HASE Masato 長谷正人 (dir.),

Bunka shakaigaku nyūmon 文化

社会学入門 (Introduction à la sociologie

culturelle), Kyōto, Mineruva shobō

ミネルヴァ書房.

TANAKA Daisuke 田中大介 2013

« Konbini to kafe» コンビニとカフェ

(Konbini et café), in NAKASUл Naoya 中筋

直哉 \& IGARASH Yasumasa 五十嵐泰正 (dir.),

Yoku wakaru toshi shakaigaku

よくわかる都市社会学 (Bien comprendre

la sociologie urbaine), Kyōto, Mineruva shobō ミネルヴァ書房.

TsUNEKI Teruo 常木暎生 2005

« Konbini riyō ni kansuru kenkyū (1) » コンビニ利用に関する研究 (1) (Recherche sur l'usage des konbini [1]), Kansai

daigaku shakaigakubu kiyō 関西大学 社会学部紀要 (Bulletin de la faculté de sociologie de l'université du Kansai), 36 (3) : 1-25.

\section{WsHızu Tsutomu 鷲巣力 2008}

Kōkyōkūkan to shite no konbini. Shinka suru shisutemu nijūyon jikan sanbyaku rokujū go nichi 公共空間としてのコンビニ 一進化するシステム24時間365日 (Les konbini comme espaces publics. Des systèmes en évolution permanente, 24h/24, 365 jours par an), Tokyo, Asahi shinbun shuppan 朝日新聞出版.

\section{WATANABE Hiroaki 渡邊博顕 2005} « Nikkeijin, kenshūsei/ginō jisshūsei, ryūgakusei, shūgakusei... Teichingin no gaikokujin rōdōsha ga Nippon keizai o sasaeteiru »日系人、研修生·技能実習 生、留学生、就学生…低賃金の外国人労働 者が日本経済を支えている (Descendants d'immigrants japonais, stagiaires/ internes, étudiants internationaux, élèves étrangers... Les travailleurs étrangers à bas salaire en soutien de l'économie japonaise), Ekonomisuto エコノミスト (The Economist), 8 mars 2005 : 88-89.

\section{Yamada Atsuo 山田淳夫 1996}

Kieru hon.ya. Shuppan ryūtsū ni nani ga okiteiruka? 消える本屋一出版流通に何が 起きているか (L'extinction des librairies. Que se passe-t-il dans le circuit de distribution du livre ?), Tokyo, Arumedia アルメディア. 